\title{
Análisis de la asociación entre procalcitonina y mortalidad por sepsis en menores de 18 años en una unidad de cuidados intensivos, Pasto - Colombia 2012 - 2014
}

\author{
Analysis of the association between Procalcitonin and mortality by sepsis in children under 18 years of age in an \\ intensive care unit in Pasto, Colombia between 2012 and 2014
}

Freddy Pantoja-Chamorro ${ }^{*}$ orcid.org/0000-0002-2856-9624

Liliana Mazzillo-Vega' ${ }^{1}$ orcid.org/0000-0002-1085-4101

Uriel Palacios-Barahona² ${ }^{2}$ orcid.org/0000-0002-3740-0353

1 Hospital Infantil los Ángeles. San Juan de Pasto, Colombia

2 Centro de Evaluación de Tecnologías en salud (CETES). Medellín, Colombia

Fecha de recepción: Julio 7 - $2017 \quad$ Fecha de revisión: Noviembre 10 - $2017 \quad$ Fecha de aceptación: Diciembre 21 - 2017

Pantoja-Chamorro F, Mazzillo-Vega L, Palacios-Barahona U. Análisis de la asociación entre procalcitonina y mortalidad por sepsis en menores de 18 años en una unidad de cuidados intensivos, Pasto- Colombia 2012 - 2014. Univ. Salud. 2018;20(1):64-71. DoI: http://dx.doi.org/10.22267/rus.182001.110

\section{Resumen}

Introducción: La procalcitonina aumenta tempranamente en pacientes con sepsis, sin embargo, es necesario profundizar la relación entre sus niveles y el compromiso multiorgánico, así como sus desenlaces como la muerte. Objetivo: Determinar si existe asociación entre los valores de procalcitonina y la muerte en niños con sepsis que ingresan a la unidad de cuidado intensivo pediátrico (UCIP). Materiales y métodos: Estudio de corte transversal de pacientes menores de 18 años con sepsis que ingresaron a la UCIP del Hospital Infantil Los Ángeles, Pasto - Colombia, durante 2012 y 2014. Se analizó la relación entre el riesgo de muerte y los niveles de procalcitonina controlando por covariables clínicas y demográficas. Resultados: Se analizaron datos de 325 pacientes con sepsis, la enfermedad mostró una prevalencia de 27,3\%, de los cuales fallecieron el 23,7\% (77). La edad mediana fue 22 meses, rango intercuartil- RI (6 - 84). La mediana de procalcitonina fue de $10 \mathrm{ng} / \mathrm{mL}$, RI $(2,95$ - 38). Niños con valores $>10 \mathrm{ng} / \mathrm{mL}$ tienen un incremento del riesgo de muerte de 2 a 6 veces más comparados con aquellos que presentaron valores inferiores. Conclusión: La procalcitonina puede ser utilizada como indicador y predictor del riesgo de muerte en menores de 18 años con sepsis.

Palabras clave: Procalcitonina; supervivencia; muerte; sepsis; niño; cuidados críticos; pronóstico. (Fuente: DeCS, Bireme).

\begin{abstract}
Introduction: Procalcitonin increases early in patients with sepsis; however, it is necessary to deepen the relationship between their levels and the multi-organ commitment, as well as their outcomes such as death. Objective: To determine whether there is an association between the values of Procalcitonin and death in children with sepsis entering the pediatric intensive care unit (PICU). Materials and methods: A cross-sectional study was made with patients under 18 years of age with sepsis, who entered the PICU of Los Angeles children's Hospital in Pasto, Colombia during 2012 and 2014. The relationship between the risk of death and the levels of Procalcitonin controlled by clinical and demographic covariates was analyzed. Results: Data from 325 patients with sepsis was analyzed. The disease showed a prevalence of $27.3 \%$, from which $23.7 \%$ (77) died. The median age was 22 months, interquartile-RI range (6-84). The median Procalcitonin was $10 \mathrm{ng} / \mathrm{mL}$, RI (2.95-38). Children with values $>10 \mathrm{ng} / \mathrm{mL}$ have an increase in the risk of death from 2 to 6 times more, compared to those who presented lower values. Conclusion: Procalcitonin can be used as an indicator and predictor of the risk of death in children under 18 with sepsis.
\end{abstract}

*Autor de correspondencia 
Keywords: Procalcitonin; survival; death; sepsis; child; critical care; prognosis. (Source: DeCS, Bireme).

\section{Introducción}

La mortalidad infantil es una de las prioridades en la salud pública a nivel mundial, para 2012 según la Organización Mundial de la Salud (OMS) murieron 56 millones de personas de los cuales 6,6 millones fallecieron antes de cumplir los 5 años(1). Según las Naciones Unidas, la tasa promedio de mortalidad infantil a nivel mundial es de 49,4 por mil nacidos vivos; de esas muertes prematuras, más del $50 \%$ se pueden evitar con acciones de prevención e intervención básicas y oportunas $^{(1)}$. En Colombia mueren cerca de 8.000 niños al año y la mayoría de las defunciones de los menores de un año se concentra en las zonas rurales, donde la tasa de mortalidad no ajustada asciende a 14 defunciones por cada mil nacidos vivos, registro que disminuye en las cabeceras municipales a menos de 11 muertes por cada mil nacidos vivos(2). La mayor proporción de muertes infantiles se debe a infecciones como la neumonía que genera el $18 \%$ de todas las muertes infantiles, diarrea $(11 \%)$ y paludismo $(7 \%)^{(1)}$. Estas infecciones localizadas pueden avanzar complicándose con infecciones multisistémicas graves como la sepsis, aumentando la probabilidad de muerte.

La sepsis es un problema común en pacientes que ingresan a los servicios de urgencias y cuidado intensivo, por esto que es importante que cuando se inician los estudios clínicos y de laboratorio enfocados a determinar la presencia de sepsis, se hagan de manera temprana para que el tratamiento se enfoque de forma oportuna y eficaz, permitiendo que los niveles de morbilidad y mortalidad disminuyan considerablemente ${ }^{(3)}$. Detectar el estado de gravedad de los pacientes que ingresan con sepsis no es fácil, ya que los hallazgos clínicos pueden ser mínimos o inespecíficos en fases iniciales de la enfermedad por ello se han planteado marcadores de laboratorio para determinarla, sin embargo poco se sabe de estos marcadores que permitan establecer el pronóstico de vida luego del ingreso de un niño con sepsis a la unidad de cuidados intensivos ${ }^{(4)}$.
La procalcitonina es una proteína precursora de la calcitonina, que aumenta sus niveles tempranamente en pacientes con sepsis(5). En algunos estudios se ha encontrado que niveles elevados ( $>0,5 \mathrm{a} \geq 2,0 \mathrm{ng} / \mathrm{ml}$ ) tienen un valor predictivo positivo en el diagnóstico de sepsis y choque séptico, siendo una señal de alarma que indica alto riesgo de disfunción de órganos debido a la inflamación sistémica, de igual forma se ha reportado que niveles muy bajos o negativos (< $0,25 \mathrm{a}<0,5 \mathrm{ng} / \mathrm{mL}$ ) tienen un alto valor predictivo negativo permitiendo descartar inflamación sistémica grave o sepsis(6). Por lo tanto se ha postulado que niveles $\geq 10 \mathrm{ng} / \mathrm{mL}$ de procalcitonina en pacientes con sepsis, están relacionados con la gravedad de la enfermedad y la probabilidad de muerte(5-8).

Estos estudios han mostrado la utilidad de la procalcitonina en el diagnóstico temprano de sepsis, así como la reducción del uso de antibióticos y la estancia hospitalaria, entre otros beneficios $^{(9-13)}$. Sin embargo, no hay suficiente evidencia que establezca que la procalcitonina predice la sobrevida en población infantil(14-23). Este estudio pretende determinar si existe relación entre valores de procalcitonina y la muerte en niños con sepsis que ingresan a la unidad de cuidado intensivo pediátrico (UCIP).

\section{Materiales y métodos}

Se realizó un estudio de corte transversal de todos $(\mathrm{N}=325)$ los pacientes mayores de un mes y menores de 18 años que ingresaron a la unidad de cuidado intensivo del Hospital Infantil Los Ángeles de la ciudad de Pasto - Colombia, con diagnóstico de sepsis durante el período 2012 y 2014 y que tuvieran registro de muestra sérica de procalcitonina durante las primeras 24 horas de ingreso a la unidad. El diagnóstico de sepsis sigue la definición de las guías internacionales (24). De los 1376 pacientes que ingresaron en los años de estudio, se excluyeron aquellos pacientes en estado pos paro cardíaco, muerte cerebral, trauma, cirugía cardiovascular, pancreatitis, o quemaduras, que se hubieran presentado previo a su ingreso a la UCIP; se identificaron 376 con 
diagnóstico de sepsis de los cuales 39 presentaron registros de historia clínica incompletos, o sin reporte de procalcitonina en las primeras 24 horas de ingreso a la UCIP y fueron remitidos a otra institución luego del ingreso a la unidad (Figura 1).

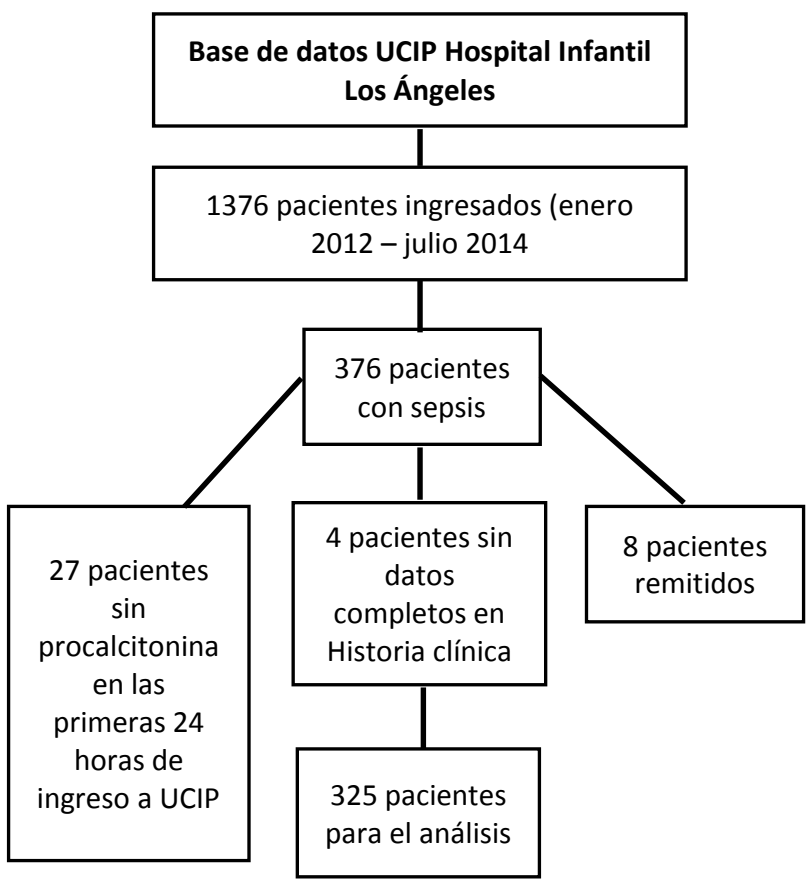

Figura 1. Flujograma de selección de los pacientes incluidos

Se recopiló información sobre los valores de procalcitonina, muerte por sepsis y algunas condiciones clínicas (estado nutricional, oportunidad de ingreso a la UCIP, infecciones asociadas a la atención en salud (IAAS), compromiso de órganos, estancia) y demográficas (edad, sexo, seguridad social). La medición de procalcitonina fue mediante VIDAS B.R.A.H.M.S procalcitonina (Biomerieux, Marcy l'Etoile, France) que tiene una sensibilidad funcional desde $0,06 \mathrm{ng} / \mathrm{mL}$ y sus resultados son analizados automáticamente en concentraciones de $\mathrm{ng} / \mathrm{mL}$.

La información fue recolectada por los investigadores extrayendo el registro de pacientes con diagnóstico de sepsis directamente de una base de datos de la unidad de cuidado intensivo para luego hacer revisión de cada historia clínica sistematizada, de esta manera se evita el sobre registro o subregistro de los pacientes con el diagnostico de sepsis.

No se excluyeron variables ni se imputaron datos, sin embargo, solo un investigador chequeó los diagnósticos en las historias clínicas, verificó valores de procalcitonina y otros datos clínicos o de laboratorio que pudieran estar errados; se aplicó un instrumento para la recolección de datos y se realizó una prueba piloto.

Las variables cualitativas se resumieron en forma de frecuencias absolutas y relativas y las cuantitativas con medidas de tendencia central y dispersión. Se realizó un modelo multivariado de regresión logística para determinar el riesgo de morir (Odds Ratio: OR) entre pacientes con niveles bajos de procalcitonina frente a valores altos, ajustados por condiciones clínicas y demográficas. Todos los análisis se realizaron con un $95 \%$ de confianza en el software SPSS $^{\circledR}$, versión 21.

\section{Consideraciones éticas}

El proyecto fue aprobado por el Comité de Ética de la Universidad CES y el Hospital Infantil Los Ángeles. Este estudio se clasificó como "Sin Riesgo" de acuerdo con el artículo 11 de la Resolución 8430 de 1993.

\section{Resultados}

La población infantil con sepsis $(\mathrm{N}=325)$ representa el $27,3 \%$ de los pacientes que ingresaron a la unidad de cuidado intensivo pediátrico. En esta población se observaron 77 muertes $(27,3 \%)$. Las mujeres representaron el $46 \%$; la edad mediana fue de 22 meses, rango intercuartil- RI (6 - 84), por grupos de edad, el $63 \%$ de los pacientes eran lactantes, un $17 \%$ escolares, $13 \%$ adolescentes y $7 \%$ preescolares, la mediana de los días de estancia fue de 5 días, RIC (3-10), el principal foco de infección fue el pulmón con un 52,6\% (171), los pacientes con desnutrición comprendieron el 39\% (127). La mediana de procalcitonina en el grupo de fallecidos fue de $33 \mathrm{ng} / \mathrm{mL}$ RI (8 -109) y la de los supervivientes fue de $6,5 \mathrm{ng} / \mathrm{mL}$ RI (2,5 - 4). (Tabla 1.) 
Tabla 1. Distribución de las características demográficas y clínicas de los pacientes en UCIP con sepsis según estado vital, Pasto 2012-2014

\begin{tabular}{|c|c|c|c|c|c|c|}
\hline & & & & Muerte & & \\
\hline & Variable & & Si (n & & No $(n=24$ & \\
\hline & & & $\mathbf{n} *$ & $\% *$ & $\mathbf{n} *$ & \%* \\
\hline Procalcitonina & Baja & $<5 \mathrm{ng} / \mathrm{mL}$ & 13 & 16,9 & 111 & 44,8 \\
\hline categorizada & Moderada & $5-10 \mathrm{ng} / \mathrm{mL}$ & 9 & 11,7 & 34 & 13,7 \\
\hline & Alta & $10-50 \mathrm{ng} / \mathrm{mL}$ & 26 & 33,8 & 62 & 25 \\
\hline & Muy alta & $>50 \mathrm{ng} / \mathrm{mL}$ & 29 & 37,7 & 41 & 16,5 \\
\hline Procalcitonina** & & & 10 & & $(2,95-38)$ & \\
\hline Variables clínicas & & & & & & \\
\hline Desnutrición & No & & 37 & 48,1 & 161 & 64,9 \\
\hline & $\mathrm{Si}$ & & 40 & 51,9 & 87 & 35,1 \\
\hline Ingreso oportuno a la & $\mathrm{Si}$ & & 30 & 39 & 163 & 65,7 \\
\hline unidad & No & & 47 & 61 & 85 & 34,3 \\
\hline Infección asociada a la & No & & 65 & 84,4 & 226 & 91,1 \\
\hline atención en salud & $\mathrm{Si}$ & & 12 & 15,6 & 22 & 8,9 \\
\hline Origen de la infección & Articular & & 7 & 9,1 & 13 & 5,2 \\
\hline & Pulmón & & 43 & 55,8 & 128 & 51,6 \\
\hline & Urinario & & 1 & 1,3 & 10 & 4 \\
\hline & Oncología & & 4 & 5,2 & 6 & 2,4 \\
\hline & Intestinal & & 15 & 19,5 & 80 & 32,3 \\
\hline & SNC & & 7 & 9,1 & 11 & 4,4 \\
\hline Sepsis grave & No & & 2 & 2,6 & 74 & 29,8 \\
\hline & $\mathrm{Si}$ & & 75 & 97,4 & 174 & 70,2 \\
\hline Choque séptico & No & & 11 & 14,3 & 188 & 75,8 \\
\hline & $\mathrm{Si}$ & & 66 & 85,7 & 60 & 24,2 \\
\hline Compromiso de & No & & 18 & 23,4 & 104 & 41,9 \\
\hline pulmón & $\mathrm{Si}$ & & 59 & 76,6 & 144 & 58,1 \\
\hline Compromiso de & No & & 37 & 48,1 & 212 & 85,5 \\
\hline corazón & $\mathrm{Si}$ & & 40 & 51,9 & 36 & 14,5 \\
\hline Compromiso de riñón & No & & 35 & 45,5 & 216 & 87,1 \\
\hline & $\mathrm{Si}$ & & 42 & 54,5 & 32 & 12,9 \\
\hline Coagulopatía & No & & 24 & 31,2 & 192 & 77,4 \\
\hline & $\mathrm{Si}$ & & 53 & 68,8 & 56 & 22,6 \\
\hline Compromiso de & No & & 23 & 29,9 & 178 & 71,8 \\
\hline hígado & $\mathrm{Si}$ & & 54 & 70,1 & 70 & 28,2 \\
\hline Órganos afectados & menos de 3 & & 54 & 70,1 & 46 & 18,5 \\
\hline & 3 o más & & 202 & 81,5 & 23 & 29,9 \\
\hline Estancia ** & & & 5 & & $(3-10)$ & \\
\hline Variables demográfi & & & & & & \\
\hline Seguridad social & Contributivo & & 3 & 3,90 & 22 & 8,9 \\
\hline & Subsidiado & & 74 & 96,10 & 226 & 91,1 \\
\hline Sexo & Masculino & & 44 & 57,10 & 130 & 52,4 \\
\hline & Femenino & & 33 & 42,90 & 118 & 47,6 \\
\hline Procedencia & Otros & & 24 & 31,20 & 97 & 39,1 \\
\hline & Pasto & & 13 & 16,90 & 48 & 19,4 \\
\hline & Pacífico & & 20 & 26,00 & 56 & 22,6 \\
\hline & Putumayo & & 20 & 26,00 & 47 & 19 \\
\hline Edad categorizada & Adolescente & & 10 & 13,00 & 31 & 12,5 \\
\hline & Lactante & & 58 & 75,30 & 148 & 59,7 \\
\hline & Preescolar & & 3 & 3,90 & 21 & 8,5 \\
\hline & Escolar & & 6 & 7,80 & 48 & 19,4 \\
\hline Edad $^{* *}$ & & & 22 & & $(6-84)$ & \\
\hline
\end{tabular}

*Para variables cuantitativas no normales mediana y rango intercuartílico

**Variables no normales ante la prueba de Kolmogorov-Smirnov $\mathrm{P}<0,05$

Se observó que los valores de procalcitonina (10 $49 \mathrm{ng} / \mathrm{mL}$ ) incrementan la probabilidad morir 3,58 veces respecto a los valores $\leq 4 \mathrm{ng} / \mathrm{ml}$; los valores $\geq 50 \mathrm{ng} / \mathrm{ml}$ incrementan el riesgo 5,87 veces respecto a valores $\leq 4 \mathrm{ng} / \mathrm{ml}$. Los pacientes con desnutrición tuvieron la probabilidad 2 veces 
más de fallecer que los pacientes sin desnutrición $\mathrm{OR}=2,00$ (IC 1,19 - 3,36). Se identificó que los pacientes con sepsis grave, compromiso pulmonar, cardíaco, hepático, renal $\mathrm{y}$ coagulopatía tuvieron mayor posibilidad de morir con respecto a los que no tenían dichas condiciones (Tabla 1).

Luego de ajustar el OR se observó que la variable procalcitonina perdió significancia estadística. Se ajustó el siguiente modelo teniendo en cuenta variables con mayor significancia clínica y con mayor representación dentro del escenario de un paciente con sepsis frente a la muerte. Se observó que al excluir el compromiso hepático y la coagulopatía los valores altos de procalcitonina $(10-50 \mathrm{ng} / \mathrm{mL})$ incrementan el riesgo de morir 2,8 veces respecto a los que no prsentan estos (IC 1,02 - 7,92); los valores muy altos (>50 ng/mL) incrementan el riesgo 3,4 veces respecto a los que no los presentaron (IC 1,08-10,96) (Tabla 2).

Tabla 2. Factores asociados a la muerte en UCIP

\begin{tabular}{|c|c|c|c|c|c|c|c|c|c|c|c|}
\hline \multicolumn{2}{|c|}{ Variable } & \multirow{2}{*}{\begin{tabular}{|l|}
$\begin{array}{l}\text { OR } \\
\text { Crudo }\end{array}$ \\
3,58
\end{tabular}} & \multicolumn{2}{|c|}{ IC (95\%) } & \multirow{2}{*}{$\begin{array}{r}\text { Valor } \mathbf{p} \\
0\end{array}$} & \multicolumn{2}{|c|}{ Variable } & \multirow{2}{*}{$\begin{array}{l}\begin{array}{l}\text { OR } \\
\text { Ajustado }\end{array} \\
2,84\end{array}$} & \multicolumn{2}{|c|}{ IC (95\%) } & \multirow{2}{*}{$\begin{array}{r}\text { Valor } \mathbf{p} \\
0,05\end{array}$} \\
\hline $\begin{array}{l}\text { Procalcitonina } \\
\text { categorizada }\end{array}$ & $\begin{array}{l}\text { Alta 10-50 } \\
\mathrm{ng} / \mathrm{mL}\end{array}$ & & 1,72 & 7,47 & & $\begin{array}{l}\text { Procalcitonina } \\
\text { categorizada }\end{array}$ & $\begin{array}{l}\text { Alta 10-50 } \\
\mathrm{ng} / \mathrm{mL}\end{array}$ & & 1,02 & 7,92 & \\
\hline & $\begin{array}{l}\text { Muy alta }>50 \\
\mathrm{ng} / \mathrm{mL}\end{array}$ & 6,04 & 2,87 & 12,73 & 0 & & $\begin{array}{l}\text { Muy alta }>50 \\
\mathrm{ng} / \mathrm{mL}\end{array}$ & 3,44 & 1,08 & 10,96 & 0,04 \\
\hline \multirow[t]{2}{*}{ Desnutrición } & No & & & & & Desnutrición & No & & & & \\
\hline & $\mathrm{Si}$ & 2 & 1,19 & 3,36 & 0,01 & & $\mathrm{Si}$ & 3,24 & 1,37 & 7,64 & 0,01 \\
\hline \multirow[t]{2}{*}{ Choque séptico } & No & & & & & Choque séptico & No & & & & \\
\hline & $\mathrm{Si}$ & 18,8 & 9,32 & 37,91 & 0 & & $\mathrm{Si}$ & 9,03 & 3,72 & 21,95 & 0 \\
\hline \multirow[t]{2}{*}{$\begin{array}{l}\text { Compromiso de } \\
\text { riñón }\end{array}$} & No & & & & & Compromiso de & No & & & & \\
\hline & $\mathrm{Si}$ & 8,1 & 4,53 & 14,5 & 0 & & $\mathrm{Si}$ & 2,38 & 0,96 & 5,91 & 0,06 \\
\hline
\end{tabular}

\section{Discusión}

Este tipo de estudio únicamente permite explorar relaciones, considerando su diseño se debe tener en cuenta que existen algunas limitaciones importantes: no se hizo muestreo, ya que se intentó tomar la mayor cantidad de pacientes durante los dos años y medio evaluados y es un estudio en donde se extrajo información de registros de historias clínicas de forma retrospectiva existiendo el riesgo de caer en sesgos de información. Sólo se evaluó el valor de procalcitonina al ingreso del paciente por ello no se pudo observar el comportamiento durante el tiempo de hospitalización. No se comparó la procalcitonina con otro marcador inflamatorio o escalas de gravedad. La cantidad considerable de pacientes pediátricos estudiados es una fortaleza del trabajo, además de ser el primer estudio analítico en la región que se lleva a cabo con este marcador que ha cobrado cada vez más importancia y que se utiliza rutinariamente por el personal de salud de la institución.
Este estudio describe la serie más grande de pacientes con sepsis y valores de procalcitonina asociados a mortalidad en niños mayores de 1 mes reportada en Latinoamérica. Al realizar la búsqueda de literatura existente el estudio de Gómez-Rivera et al.(7), quienes evaluaron 22 pacientes con sepsis observando que la mortalidad al séptimo día se presentó con mayor frecuencia en los pacientes que presentaron procalcitonina $>10 \mathrm{ng} / \mathrm{mL}$. Hatherill et al.(25), observaron en 75 niños con sepsis niveles de procalcitonina de $82 \mathrm{ng} / \mathrm{mL}$ (supervivientes) vs. $273 \mathrm{ng} / \mathrm{mL}$ (no supervivientes) (IC 95\%: (0,59$0,880)$; aquí se estudió la relación de niveles de procalcitonina, interleuquina 10 y el factor de necrosis tumoral con la mortalidad, concluyendo que niveles elevados de estos tres marcadores séricos se asocian con la mortalidad en niños con choque séptico. Casado-Flores et al.(26), en un estudio de 80 niños con sepsis, reportaron que el valor de la mediana de procalcitonina al ingreso, fue significativamente más alto en los no 
sobrevivientes, encontrando diferencia estadística significativa en los sobrevivientes (49,6 vs. $16,4 \mathrm{ng} / \mathrm{mL}$ ) ( $\mathrm{p}=0,008)$, consecuente con los hallazgos del presente trabajo (33 vs 6 ng/mL) (IC: 95\%: 2,95-38) respectivamente.

Jensen et $a l .(3)$, en un estudio de población heterogénea con un rango de edad de 11 meses a 92 años evaluaron 472 pacientes críticos, encontraron que valores de procalcitonina $>20$ $\mathrm{ng} / \mathrm{mL}$ (IC 95\%: 1,9-8,4) son predictores independientes de mortalidad. Los reportes de prevalencia de sepsis y letalidad por sepsis coincidieron con los valores reportados en otras unidades de cuidado crítico, en donde se muestran cifras de $23 \%$ a $52 \%$ para la prevalencia y de $18 \%$ a $56 \%$ para la letalidad(27-29). De esta manera y teniendo en cuenta el análisis bivariado se evidenció que los valores altos de procalcitonina (mayores de $10 \mathrm{ng} / \mathrm{ml}$ ) en los pacientes con sepsis se relacionan con muerte, sin embargo, no se debe dejar de considerar algunas covariables que pudieran influir en la muerte. Es importante resaltar que existen otros factores de riesgo que clínicamente soportan el desenlace fatal de los pacientes con procalcitonina alta como el estado de desnutrición, el ingreso tardío a la unidad de cuidado intensivo, el estado de choque séptico y el compromiso renal, como se ha evidenciado en diferentes estudios ${ }^{(30,31)}$.

Se conoce el impacto del estado nutricional frente al sistema inmune, la desnutrición está asociada con alto riesgo y severidad de infecciones por bacterias, virus, hongos y parásitos, llevando a los pacientes a estados graves de infección generando altas tasas de mortalidad(32). Cabe mencionar que las tasas de mortalidad en sepsis disminuyen considerablemente al iniciar tratamientos oportunos dentro de los que se destacan la reanimación hídrica, inicio temprano de antibióticos, vasopresores e inotrópicos por lo cual el ingreso temprano a cuidado intensivo o la remisión oportuna a un centro de mayor complejidad es vital(24). Se demostró de igual manera que la mayor incidencia de pacientes con sepsis severa se presenta en la edad de lactantes, pero así mismo se observa como estos sobreviven a la hospitalización a pesar de que su estado inmunitario está menos desarrollado en estas etapas de la vida(33).

El análisis, se evidenció que los valores altos de procalcitonina (mayores de $10 \mathrm{ng} / \mathrm{ml}$ ) en los pacientes con sepsis se relacionan con muerte, sin embargo, no se deben dejar de considerar algunas covariables que también pudieran influir en la muerte como se mencionó anteriormente: compromiso nutricional (desnutrición), choque séptico y lesión renal, este último ya demostrado como un factor independiente de muerte en los pacientes pediátricos con $\operatorname{sepsis}^{(34)}$.

\section{Conclusión}

Se considera entonces que la procalcitonina puede llegar a ser un buen marcador de muerte siempre y cuando sea usada con otros marcadores clínicos y de laboratorio en los pacientes con sepsis, llegando a ser útil en los servicios de urgencias y cuidado intensivo, sin embargo, se recomienda realizar estudios multicéntricos en donde se pueda comparar este tipo de marcadores con otros, o con escalas de gravedad de pacientes que ingresan a cuidado intensivo, inclusive considerarla como parte de una de las escalas.

\section{Agradecimientos}

Al Hospital Infantil Los Ángeles de la ciudad de Pasto, Universidad CES de Medellín y a la Universidad Mariana.

\section{Conflicto de intereses}

Ninguno declarado por los autores.

\section{Financiación}

Universidad CES y Universidad Mariana.

\section{Referencias}

1. Organización Mundial de la Salud. Reducción de la mortalidad en la niñez [Internet]. Ginebra: OMS; 2016. [citado el 6 de febrero de 2015]. Disponible en: http://www.who.int/mediacentre/factsheets/fs178/es

2. Programa de las Naciones Unidas para el Desarrollo. Objetivos de Desarrollo del Milenio: Informe 2015 [Internet]. Colombia: PNUD; 2015. [citado el 14 de mayo de 2016]. Disponible en: http://www.co.undp.org/content/colombia/es/home/ 
presscenter/articles/2015/09/28/-c-mo-le-fue-acolombia-con-los-odm-.html

3. Jensen JU, Heslet L, Jensen TH, Espersen K, Steffensen P, Tvede M. Procalcitonin increase in early identification of critically ill patients at high risk of mortality. Crit Care Med. 2006;34(10):2596-602.

4. Kibe S, Adams K, Barlow G. Diagnostic and prognostic biomarkers of sepsis in critical care. J Antimicrob Chemother. 2011;66(Suppl 2):ii33-40.

5. Valdez-Costeira R, Huerta-Romano JF. Utilidad de procalcitonina en niños con infección sistémica (primera de dos partes). Rev Mex Pediatría. 2011;78(4):147-51.

6. Meisner M. Update on procalcitonin measurements. Ann Lab Med. 2014;34(4):263-73.

7. Gómez-Rivera N, Molina-Moreno FA, García-Zarate MG, Castillo-Ramos J, García-Hernández RJ, Vázquez-Pizaña E, et al. Procalcitonina semicuantitativa (PCT-Q) como prueba diagnóstica y marcador pronóstico en niños con sepsis bacteriana. Bol Méd Hosp Infant México. 2006;63(1):8-17.

8. Han YY, Doughty LA, Kofos D, Sasser H, Carcillo JA. Procalcitonin is persistently increased among children with poor outcome from bacterial sepsis. Pediatr Crit Care Med J Soc Crit Care Med World Fed Pediatr Intensive Crit Care Soc. 2003;4(1):21-5.

9. Pundiche M, Sârbu V, Unc OD, Grasa C, Martinescu A, Bădărău V, et al. [Role of procalcitonin in monitoring the antibiotic therapy in septic surgical patients]. Chir Buchar Rom 1990. 2012;107(1):71-8.

10. Soni NJ, Samson DJ, Galaydick JL, Vats V, Huang ES, Aronson N, et al. Procalcitonin-guided antibiotic therapy: a systematic review and meta-analysis. J Hosp Med. 2013;8(9):530-40.

11. Saeed K, Dryden M, Bourne S, Paget C, Proud A. Reduction in antibiotic use through procalcitonin testing in patients in the medical admission unit or intensive care unit with suspicion of infection. J Hosp Infect. 2011;78(4):289-92.

12. Liu B, Li H, Lei Y, Zhao S, Sun M. [Clinical significance of dynamic monitoring of procalcitonin in guiding the use of antibiotics in patients with sepsis in ICU]. Zhonghua Wei Zhong Bing Ji Jiu Yi Xue. 2013;25(11):690-3.

13. Haubitz S, Mueller B, Schuetz P. Streamlining antibiotic therapy with procalcitonin protocols: consensus and controversies. Expert Rev Respir Med. 2013;7(2):14557.

14. Wang T, Wang Z, Wang L, Yan L, Wan J, Zhang S, et al. CRISPLD2 is expressed at low levels during septic shock and is associated with procalcitonin. PloS One. 2013;8(6): e65743.

15. Viallon A, Guyomarc'h S, Marjollet O, Berger C, Carricajo A, Robert F, et al. Can emergency physicians identify a high mortality subgroup of patients with sepsis: role of procalcitonin. Eur J Emerg Med Off J Eur Soc Emerg Med. 2008;15(1):26-33.

16. Su L, Meng K, Zhang $\mathrm{X}$, Wang $\mathrm{H}$, Yan $\mathrm{P}$, Jia $\mathrm{Y}$, et al. Diagnosing ventilator-associated pneumonia in critically ill patients with sepsis. Am J Crit Care Off Publ Am Assoc Crit-Care Nurses. 2012;21(6):e110-119.
17. Prkno A, Wacker C, Brunkhorst FM, Schlattmann P. Procalcitonin-guided therapy in intensive care unit patients with severe sepsis and septic shock--a systematic review and meta-analysis. Crit Care Lond Engl. 2013;17(6):R291.

18. Pettilä V, Hynninen M, Takkunen O, Kuusela P, Valtonen M. Predictive value of procalcitonin and interleukin 6 in critically ill patients with suspected sepsis. Intensive Care Med. 2002;28(9):1220-5.

19. Magrini L, Travaglino F, Marino R, Ferri E, De Berardinis B, Cardelli P, et al. Procalcitonin variations after Emergency Department admission are highly predictive of hospital mortality in patients with acute infectious diseases. Eur Rev Med Pharmacol Sci. 2013;17(Suppl 1):133-42.

20. Li Z, Wang H, Liu J, Chen B, Li G. Serum soluble triggering receptor expressed on myeloid cells- 1 and procalcitonin can reflect sepsis severity and predict prognosis: a prospective cohort study. Mediators Inflamm. 2014;2014:641039.

21. Jain S, Sinha S, Sharma SK, Samantaray JC, Aggrawal P, Vikram NK, et al. Procalcitonin as a prognostic marker for sepsis: a prospective observational study. BMC Res Notes. 2014;7:458.

22. Clec'h C, Fosse J-P, Karoubi P, Vincent F, Chouahi I, Hamza L, et al. Differential diagnostic value of procalcitonin in surgical and medical patients with septic shock. Crit Care Med. 2006;34(1):102-7.

23. Clec'h C, Ferriere F, Karoubi P, Fosse JP, Cupa M, Hoang $\mathrm{P}$, et al. Diagnostic and prognostic value of procalcitonin in patients with septic shock. Crit Care Med. 2004;32(5):1166-9.

24. Dellinger RP, Levy MM, Rhodes A, Annane D, Gerlach H, Opal SM, et al. Surviving Sepsis Campaign: international guidelines for management of severe sepsis and septic shock, 2012. Intensive Care Med. 2013;39(2):165-228.

25. Hatherill M, Tibby SM, Turner C, Ratnavel N, Murdoch IA. Procalcitonin and cytokine levels: relationship to organ failure and mortality in pediatric septic shock. Crit Care Med. 2000;28(7):2591-4.

26. Casado-Flores J, Blanco-Quirós A, Asensio J, Arranz E, Garrote JA, Nieto M. Serum procalcitonin in children with suspected sepsis: a comparison with C-reactive protein and neutrophil count. Pediatr Crit Care Med. 2003;4(2):190-5.

27. Jain S, Sinha S, Sharma SK, Samantaray JC, Aggrawal P, Vikram NK, et al. Procalcitonin as a prognostic marker for sepsis: a prospective observational study. BMC Res Notes. 2014;7:458.

28. Grozdanovski K, Milenkovic Z, Demiri I, Spasovska K, Cvetanovska M, Kirova-Urosevic V. Early prognosis in patients with community-acquired severe sepsis and septic shock: analysis of 184 consecutive cases. Prilozi. 2012;33(2):105-16.

29. Martin GS, Mannino DM, Eaton S, Moss M. The epidemiology of sepsis in the United States from 1979 through 2000. N Engl J Med. 2003;348(16):1546-54.

30. Fortenberry JD, Paden ML, Goldstein SL. Acute kidney injury in children: an update on diagnosis and treatment. Pediatr Clin North Am. 2013;60(3):669-88. 
31. Jones KDJ, Berkley JA. Severe acute malnutrition and infection. Paediatr Int Child Health. 2014;34(sup1):S129.

32. Ibrahim MK, Zambruni M, Melby CL, Melby PC. Impact of Childhood Malnutrition on Host Defense and Infection. Clin Microbiol Rev. 2017;30(4):919-71.

33. Randolph AG, McCulloh RJ. Pediatric sepsis: important considerations for diagnosing and managing severe infections in infants, children, and adolescents. Virulence. 2014;5(1):179-89.

34. Fitzgerald JC, Basu RK, Akcan-Arikan A, Izquierdo LM, Piñeres Olave BE, Hassinger $\mathrm{AB}$, et al. Acute Kidney Injury in Pediatric Severe Sepsis: An Independent Risk Factor for Death and New Disability. Crit Care Med. 2016;44(12):2241-50. 